\title{
Pancreatic sphincterotomy to manage intraductal papillary mucinous neoplasm-related recurrent pancreatitis: are we ready for a controlled trial?
}

We read with interest the study by Gonzalez et al. reporting a multicenter French experience with pancreatic sphincterotomy (PS) as treatment for intraductal papillary mucinous neoplasm (IPMN)-associated symptoms [1]. In 2017, we published a similar article on this topic including 16 patients treated in a single center [2]. In the two studies, outcomes were defined in a similar manner: a complete response was achieved when no pancreatitis episodes were observed during follow-up, whereas a partial response was defined as a reduction $>50 \%$ of pancreatitis attacks. However, quite different populations were included. In the study by Gonzalez et al. [1], only branch-duct IPMN (BD-IPMN) without worrisome features were evaluated, whereas we also included patients with main-duct IPMN unfit for surgery due to comorbidities, or patients who refused surgery. Moreover, Gonzalez et al. included patients with "post-prandial abdominal pain (typical characteristics and no associated functional disease)" [1]. However, PS should not be performed at all for "pancreatic pain" in the light of EPISOD trial results [3]. In our study, we included only patients with recurrent pancreatitis (RP) with the rigorous exclusion of those with other concomitant causes of RP [2]. It is not clear if other causes of RP were excluded in Gonzalez et al. [1]. Indeed, in $33 \%$ of their cases, a biliary sphincterotomy was also performed due to associated biliary dilatation with cholestasis or cholangitis. This selection bias could have affected their results with regard to the clinical success of PS.

In both studies, PS was performed at major or minor papilla depending on the anatomy of the ductal system. However, technical differences between the two studies should be highlighted. First, a pancreatic stent was deployed in 33\% of cases in Gonzalez et al. [1], whereas in our study, we did not place any stent following an internal policy based on the risk of stent occlusion related to the mucus passage [2]. Second, a dual sphincterotomy (biliary and pancreatic) was performed in $33 \%$ of the cases in the French study but in none of our cases $[1,2]$.

Despite the aforementioned differences in the studies' methodology and in technical practice, Gonzalez et al. [1] have reproduced our results published in 2017 [2]. Technical success was $100 \%$ in both studies. The clinical success was comparable (complete response $71 \%$ vs $69 \%$ and partial response $10 \%$ vs $19 \%$ in Gonzalez et al. and in our study, respectively) with an overall response rate of approximately $80 \%[1,2]$. However, the great limitation of the absence of control groups must be mentioned.

The procedure was safe in both experiences. Four (19\%) and one (6\%) ERCP-related mild pancreatitis were observed in Gonzalez et al. [1] and in Bernardoni et al. [2], respectively. Interestingly, in both studies, none of the BDIPMN patients developed high risk stigmata or adenocarcinoma during a long follow-up [1, 2].

Pancreatic IPMNs are often diagnosed in elderly patients affected by comorbidities who are likely to die with the cyst rather than die because of the cyst [4]. Moreover, looking at younger people, the incidence of cancer in $\mathrm{BD}$ IPMNs is rare [5]. On the other hand, pancreatic surgery is burdened by mortality and morbidity. Therefore, an effective and safe tool to treat RP in this setting of patients is a worthwhile goal. The study by Gonzalez et al. has strengthened the evidence reported in our article. Now we need a prospective multicenter controlled trial with a rigorous selection of patients and standardized technique to definitively assess the role of PS in the management of IPMNrelated pancreatitis.

\section{Competing interests}

None

The authors

Stefano Francesco Crinò, Maria Cristina Conti Bellocchi, Laura Bernardoni, Armando Gabbrielli

Digestive Endoscopy Unit, The Pancreas Institute, G.B. Rossi University Hospital, Verona, Italy

\section{Corresponding author}

Stefano Francesco Crinò, MD

Gastroenterology and Digestive Endoscopy Unit, G. B. Rossi University Hospital, P.le L.A. Scuro 10, Verona - 37134, Italy

Fax: +39-045-8124898

stefanocrino@hotmail.com

References

[1] Gonzalez JM, Lorenzo D, Ratone JP et al. Pancreatic sphincterotomy improves pain symptoms due to branch-duct intrapapillary mucinous neoplasia without worrisome features: a multicenter study. Endosc Int Open 2019; 7: E1130-E1134

[2] Bernardoni L, Crinò SF, De Conti G et al. Preliminary experience with pancreatic sphincterotomy as treatment for intraductal papillary mucinous neoplasm-associated recurrent pancreatitis. Endosc Int Open 2017; 5: E1144-E1150

[3] Cotton PB, Durkalski V, Romagnuolo J et al. Effect of endoscopic sphincterotomy for suspected sphincter of Oddi dysfunction on pain-related disability following cholecystectomy: the EPISOD randomized clinical trial. JAMA 2014; 311: 2101-2109

[4] Kwok K, Chang J, Duan L et al. Competing risks for mortality in patients with asymptomatic pancreatic cystic neoplasms: implications for clinical management. Am ] Gastroenterol 2017; 112: 1330-1336 
[5] Oyama H, Tada M, Takagi K et al. Longterm risk of malignancy in branch duct intraductal papillary mucinous neoplasms. Gastroenterology 2019: doi:10.1053/j. gastro.2019.08.032 [Epub ahead of print]
Bibliography

DOI http://dx.doi.org/10.1055/a-1024-3924

Endoscopy International Open 2019; 07: E1583-

E1584

(c) Georg Thieme Verlag KG
Stuttgart · New York elSSN 2196-9736

(ㄷ)(i) $(5)$ 